\title{
Genetic variant screening of MC3R and MC4R genes in early-onset obese children and their relatives among a Thai population: family- based study
}

S. Wannaiampikul' ${ }^{1}$ B. Phonrat ${ }^{2}$, A. Tungtrongchitr ${ }^{3}$, C. Limwongse ${ }^{4}$, N. Chongviriyaphan ${ }^{5}$, J. Santiprabhob ${ }^{6}$ and R. Tungtrongchitr ${ }^{1}$

'Department of Tropical Nutrition \& Food Science, Faculty of Tropical Medicine, Mahidol University, Bangkok, Thailand

${ }^{2}$ Department of Clinical Tropical Medicine, Faculty of Tropical Medicine, Mahidol University, Bangkok, Thailand

${ }^{3}$ Department of Parasitology, Faculty of Medicine Siriraj Hospital,

Mahidol University, Bangkok, Thailand

${ }^{4}$ Division of Molecular Genetics, Department of Research \& Development, Faculty of Medicine Siriraj Hospital, Mahidol University, Bangkok, Thailand

${ }^{5}$ Department of Pediatrics, Faculty of Medicine Ramathibodi Hospital, Mahidol University, Bangkok, Thailand

${ }^{6}$ Division of Endocrinology \& Metabolism, Department of Pediatrics,

Faculty of Medicine Siriraj Hospital, Mahidol University, Bangkok, Thailand

Corresponding author: R. Tungtrongchitr

E-mail: rungsunn.tun@mahidol.ac.th

Genet. Mol. Res. 14 (4): 18090-18102 (2015)

Received August 2, 2015

Accepted October 18, 2015

Published December 22, 2015

DOI http://dx.doi.org/10.4238/2015.December.22.35

ABSTRACT. MC3R (melanocortin-3 receptor) and MC4R (melanocortin-4 receptor) play important roles in energy homeostasis. Severe early-onset obesity, known as monogenic obesity when it is the consequence of a mutation in a single-gene product, may result when energy homeostasis is disrupted. The purpose of our study was to screen for variations of the 
MC3R and MC4R genes and observe the mode of inheritance of variations in affected families. We used polymerase chain reaction and direct sequencing to analyze the 11 early-onset obese children (probands) with their 71 family members, together with DNA from 100 healthy subjects used as controls. No novel mutations were found in the MC3R gene. Two previously described polymorphisms, rs3746619 and rs3827103, were detected in the MC3R gene. It was not associated with any obesity-related phenotypes. Three heterozygous variations of the MC4R gene were detected in 3 of 11 probands. The rs 34114122 and rs 61741819 variations have previously been reported, but rs 182455344 was novel. Moreover, each MC4R variant was also found in a number of family members, indicating that this molecular analysis of a family-based study showed an autosomal dominant pattern. Our study indicated that MC4R variations in early-onset obese Thai children were found, and transmission of these variations in each family is in the dominant pattern. These variants could possibly contribute to a genetic influence of early-onset obesity in Thais. There is no evidence of any association between MC3R variations and obesity.

Key words: Early-onset obesity; Thai family-based study; MC3R variations; MC4R variations

\section{INTRODUCTION}

The worldwide prevalence of overweight and obesity has risen considerably over the past decades, $27.5 \%$ for adults, and $47.1 \%$ for children ( $\mathrm{Ng}$ et al., 2014). Thailand reports a similar increase in prevalence of overweight and obesity among school-aged children, roughly 2.5fold between 1995 and 2009 (Yamborisut et al., 2014). The World Health Organization (WHO) has recognized obesity as a burden disease because of its physical and psychosocial health consequences. The alarming increase in obesity rates has resulted in a growing interest in the factors that contribute to this disease. The increased consumption of energy-dense fast food and sugary drinks, physical inactivity, and increased screen time are the environmental factors. In addition to these, genetic factors have been of interest. Twin studies found that genetics might account for $40-90 \%$ of human body mass index (BMI) variation (Hjelmborg et al., 2008). A familial risk study discovered that children with parents or siblings with a BMI of $\geq 40 \mathrm{~kg} / \mathrm{m}^{2}$ had a five times higher risk of becoming obese compared to individuals with normal-weight relatives (Lee et al., 1997). The leptin-melanocortin pathway plays a key role in the regulation of food intake and energy expenditure (Schwartz et al., 2000). Studies of the genetic factors causing monogenic obesity are often associated with the leptin-melanocortin pathway (Schwartz et al., 2000; Oswal and Yeo, 2007). It has been suggested that single-gene mutations of this pathway result in a deficiency of critical molecules and/or a disruption of this system, which may lead to severe early-onset obesity. The main genes that play a role in monogenic obesity are melanocortin-3 receptor (MC3R) and melanocortin-4 receptor (MC4R) (Rankinen and Bouchard, 2006). Both MC3R and MC4R are G-protein coupled receptors that mediate the anorectic response by the signal from an agonist known as alpha-melanocyte-stimulating hormone (a-MSH) (List and Habener, 2003). A range of evidence has connected early-onset obesity with MC3R and MC4R variants, and together they suggest that the condition is related to MC3R and MC4R dysfunctions without physical, hormonal, 
or developmental consequences (Vaisse et al., 1998; Yeo et al., 1998; Farooqi et al., 2003a).

MC3R has a dominant role in inhibiting energy storage and reducing food efficiency-the body's ability to convert food to adiposity (Chen et al., 2000). The prevalence of MC3R variants in severely obese subjects of European descent was nearly 2\% (Calton et al., 2009; Mencarelli et al., 2011). To date, more than 20 MC3R variants have been identified (Mencarelli et al., 2011). MC3R mutations are unlikely to result in an autosomal dominant form of monogenic obesity given the lack of strong co-segregation in family studies (Lee, 2009). MC4R mutation is the most common monogenic form of obesity in humans, accounting for up to $6 \%$ of childhood and adult obesity (Farooqi et al., 2003b). The first report of an MC4R mutation associated with obesity was published in 1998 (Vaisse et al., 1998; Yeo et al., 1998). Since then, more than 165 mutations including deletions, insertions, and point mutations have been found in the MC4R gene (Van Den Berg et al., 2011; Hinney et al., 2013). Family-based studies suggest co-dominant inheritance with incomplete expressivity and penetrance in cases of MC4R mutations (Farooqi and O'rahilly, 2008; Santini et al., 2009). The objectives of this family-based study were to identify the variations of MC3R and MC4R genes and observe the mode of inheritance in Thai children with early-onset obesity and their family members.

\section{MATERIAL AND METHODS}

\section{Study subjects}

Participants were recruited from the outpatient pediatric clinic of the Faculty of Medicine, Siriraj Hospital and Faculty of Medicine Ramathibodi Hospital, Bangkok, Thailand. The study consisted of 11 probands and 71 family members. The probands were between 8-20 years old with a percentage weight for height greater than $140 \%$, using National Growth References for Thai children under 20 years of age, 1999 (Chuenta et al., 2015). All probands had a history of early-onset obesity, as well as obese family members within at least two generations. Participants with conditions associated with obesity including Cushing syndrome, hypothyroidism, pseudohypoparathyroidism, and those with genetic disorders such as Prader-Willi syndrome and Bardet-Biedl syndrome, or those receiving hormonal/medical treatment that could contribute to obesity were excluded. Out of 71 family members, 51 had a BMI greater than $25 \mathrm{~kg} / \mathrm{m}^{2}$ according to the WHO BMI classification. These 51 subjects were classified as affected family members (overweight or obese). Twenty family members had a BMI lower than $25 \mathrm{~kg} / \mathrm{m}^{2}$, and these were classified as unaffected family members.

DNA samples from 100 individuals with non-obese, non-diabetic, and non-inherited metabolic disorders, who participated in the gene bank project at the Division of Molecular Genetics, Faculty of Medicine Siriraj Hospital, Mahidol University, were used as a control group to analyze the minor allele frequency (MAF) of each variant in the Thai population.

\section{Anthropometric and blood pressure measurements}

Standard techniques for anthropometric measurements were used. Weight was measured using a calibrated beam balance (Detecto ${ }^{\circledR}$, Webb City, MO, USA), and height was measured by a vertical-measuring rod (Microtoise, Poissy, France). The BMI was calculated as weight in kilograms divided by height in meters squared $\left(\mathrm{kg} / \mathrm{m}^{2}\right)$. Waist circumference was assessed at the midpoint between the lower border of ribs and the upper border of the pelvis. Blood pressure was recorded twice in a seated position after a 10-min rest. 


\section{Laboratory measurements}

Blood samples $(10 \mathrm{~mL})$ were taken after $12 \mathrm{~h}$ of fasting. Total cholesterol, high-density lipoprotein cholesterol (HDL-C), low-density lipoprotein cholesterol (LDL-C), triglycerides (TG), fasting blood glucose, hemoglobin $\mathrm{A} 1 \mathrm{c}(\mathrm{HbA} 1 \mathrm{c})$, and insulin were processed by using an automatic analyzer (Cobas Integra ${ }^{\circledR} 800$, Roche, Mannheim, Germany). The homeostatic model assessment of insulin resistance (HOMA-IR) was calculated as fasting blood glucose in mM multiplied by insulin in $\mathrm{pM}$ and divided by 22.5. Serum $\alpha$-MSH levels were measured using a commercial human ELISA kit from My Biosource Inc. (San Diego, CA, USA).

\section{Polymerase chain reaction (PCR) and genotyping}

Genomic DNA was extracted from peripheral blood leukocytes in EDTA-treated whole blood using a Flexi Gene DNA kit (Qiagen, Hilden, Germany). Genotyping by the DNA sequencing method for the coding region of MC3R and MC4R genes was carried out by PCR amplification using three pairs of primers for MC3R and four pairs for MC4R (Table 1). DNA sequence traces were evaluated individually by aligning and comparing them with human sequences available in GenBank. The variants were confirmed by sequencing anti-sense strands.

\begin{tabular}{|c|c|c|c|c|}
\hline Gene & Primer & Primer $\left(5^{\prime} \rightarrow 3^{\prime}\right)$ & PCR product size (bp) & $\mathrm{Ta}\left({ }^{\circ} \mathrm{C}\right)$ \\
\hline \multirow[t]{6}{*}{ MC3R } & \multirow[t]{2}{*}{ MC3R_I } & Forward: ТСТСТСТАСССТССССАТСС & \multirow[t]{2}{*}{498} & \multirow[t]{2}{*}{65} \\
\hline & & Reverse: CCTCGAAGGTCAGGTAGTCG & & \\
\hline & \multirow[t]{2}{*}{ MC3R_II } & Forward: CCGACATGCTGGTAAGTGTG & \multirow[t]{2}{*}{467} & \multirow[t]{2}{*}{65} \\
\hline & & Reverse: CATGCATGAGTGTTGCTGTG & & \\
\hline & \multirow[t]{2}{*}{ MC3R_III } & Forward: AATGGTCATTGTGTGCCTCA & \multirow[t]{2}{*}{471} & \multirow[t]{2}{*}{62} \\
\hline & & Reverse: CGTGGATGGAAAGTCAAAAGT & & \\
\hline \multirow{8}{*}{ MC4R } & \multirow{2}{*}{ MC4R_I } & Forward: TGGGATTGGTCAGAAGGAAG & \multirow[t]{2}{*}{380} & \multirow[t]{2}{*}{62} \\
\hline & & Reverse: TCCAGAGCTTGACATGGAGTT & & \\
\hline & \multirow[t]{2}{*}{ MC4R_II } & Forward: GAGAACAAGAAAGCAAAGAGCA & \multirow[t]{2}{*}{390} & \multirow[t]{2}{*}{65} \\
\hline & & Reverse: GCACCCTCCATCAGAGTAGC & & \\
\hline & \multirow[t]{2}{*}{ MC4R_III } & Forward: TTTCAGACAGATAAAGACTTG & \multirow[t]{2}{*}{618} & \multirow[t]{2}{*}{51} \\
\hline & & Reverse: CCAGATACAACTTATGATGAT & & \\
\hline & \multirow[t]{2}{*}{ MC4R_IV } & Forward: TTTACTATCTTCTATGCTCTC & \multirow[t]{2}{*}{684} & \multirow[t]{2}{*}{51} \\
\hline & & Reverse: TTACACAATGGATATTCTCAA & & \\
\hline
\end{tabular}

$\mathrm{PCR}=$ polymerase chain reaction; $\mathrm{Ta}=$ annealing temperature.

\section{Ethics approval}

This study was approved by the Ethics Committees of the Faculty of Medicine Siriraj Hospital, Faculty of Medicine Ramathibodi Hospital, and Faculty of Tropical Medicine, Mahidol University. Informed consent was obtained from participants older than 18 years old. For younger participants, informed consent was obtained from the children as well as parents.

\section{Statistical analysis}

Descriptive values are reported as median with interquartile range (IQR). The statistically significant differences of anthropometric and biochemical parameters between affected and unaffected family members were tested by the Mann-Whitney U-test. Data were analyzed using Statistical Package for Social Science version 16.0 (SPSS, Chicago, USA), and Minitab version 14.0 (New South Wales, Australia) for Windows. A P value $<0.05$ was considered to be statistically significant. 


\section{RESULTS}

This study analyzed MC3R and MC4R variants in early-onset obese children and their family members. The anthropometric and biochemical results of all probands and their family members are shown in Tables 2 and 3.

Table 2. Clinical profiles and biochemical data of 11 probands.

\begin{tabular}{|c|c|c|c|c|c|c|c|c|c|c|c|c|c|c|c|c|}
\hline Family & Gender & $\begin{array}{l}\text { er Age } \\
\text { (years) }\end{array}$ & $\begin{array}{c}\text { Age at onset } \\
\text { of obesity } \\
\text { (years) }\end{array}$ & $\begin{array}{l}\text { Percentage } \\
\text { weight for } \\
\text { height (\%) }\end{array}$ & $\begin{array}{c}\text { Weight } \\
(\mathrm{kg})\end{array}$ & $\begin{array}{c}\text { BMl } \\
\left(\mathrm{kg} / \mathrm{m}^{2}\right)\end{array}$ & $\begin{array}{l}\text { WC } \\
(\mathrm{cm})\end{array}$ & $\begin{array}{l}\text { Fasting } \\
\text { glucose } \\
(\mathrm{mg} / \mathrm{dL})\end{array}$ & $\begin{array}{c}\mathrm{HbA1c} \\
(\%)\end{array}$ & $\begin{array}{c}\text { Fasting } \\
\text { insulin } \\
(\mu \mathrm{U} / \mathrm{mL})\end{array}$ & HOMA-IR & $\begin{array}{l}\text { LDL-C } \\
\text { (mg/dL) }\end{array}$ & $\begin{array}{l}\mathrm{HDL}-\mathrm{C} \\
(\mathrm{mg} / \mathrm{dL})\end{array}$ & $\begin{array}{c}\mathrm{TG} \\
(\mathrm{mg} / \mathrm{dL})\end{array}$ & $\begin{array}{c}\mathrm{BP} \\
(\mathrm{mmHg})\end{array}$ & $\begin{array}{l}\alpha-M S H \\
\text { (pg/mL) }\end{array}$ \\
\hline A & $\mathrm{F}$ & 15 & 5 & 270 & 135 & 52.5 & 136 & 78 & 5.6 & 24.5 & 4.71 & 121 & 60 & 91 & $120 / 63$ & 10.5 \\
\hline B & $\mathrm{F}$ & 9 & $<1$ & 289 & 88 & 47.6 & 11 & 74 & 5.4 & 20.9 & 3.82 & 92 & 20 & 73 & $20 / 80$ & 42.9 \\
\hline C & M & 19 & 5 & 228 & 155 & 44.8 & 146 & 129 & 7.4 & 56.0 & 17.82 & 118 & 60 & 93 & $169 / 87$ & 22.6 \\
\hline D & M & 11 & $<1$ & 243 & 129 & 47.6 & 130 & 79 & 6.1 & 70.7 & 13.79 & 103 & 55 & 57 & $120 / 77$ & 40.3 \\
\hline E & $\mathrm{F}$ & 15 & 3 & 203 & 100 & 39.9 & 113 & 82 & 5.6 & 17.9 & 3.63 & 106 & 37 & 94 & $129 / 79$ & 24.2 \\
\hline $\mathrm{F}$ & M & 13 & 4 & 151 & 65 & 27.3 & 90 & 78 & 5.9 & 24.6 & 4.74 & 101 & 60 & 69 & $134 / 65$ & 20.0 \\
\hline G & $\mathrm{F}$ & 13 & 3 & 208 & 123 & 42.7 & 106 & 89 & 5.7 & 93.0 & 20.44 & 138 & 44 & 141 & $114 / 50$ & 50.7 \\
\hline $\mathrm{H}$ & M & 14 & 5 & & & 58.5 & 160 & 86 & 6.2 & 4.1 & 0.87 & 156 & 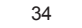 & 111 & $137 / 77$ & 81.5 \\
\hline I & M & 10 & 5 & 305 & 139 & 56.2 & 150 & 94 & 7.3 & 34.9 & 8.10 & 75 & 37 & 61 & $112 / 71$ & 40.3 \\
\hline $\mathrm{J}$ & M & 12 & 2 & 240 & 98 & 42.5 & 118 & 88 & 5.9 & 40.9 & 8.88 & 107 & 36 & 122 & $117 / 68$ & 24.2 \\
\hline K & M & 13 & 4 & 191 & 111 & 37.9 & 105 & 80 & 4.7 & 47.8 & 9.44 & 120 & 37 & 182 & $142 / 78$ & 211.1 \\
\hline
\end{tabular}

$\mathrm{BMI}=$ body mass index; $\mathrm{WC}=$ waist circumference; WHR = waist-to-hip ratio; HbA1c = hemoglobin A1c; HOMA-IR = homeostatic model assessment for insulin resistance; LDL-C = low-density lipoprotein cholesterol; HDL-C = high-density lipoprotein cholesterol; TG = triglyceride; $\mathrm{BP}=$ blood pressure; $\alpha-\mathrm{MSH}$ = serum alpha-melanocyte-stimulating hormone.

Table 3. Characteristics of unaffected and affected family members.

\begin{tabular}{|c|c|c|c|}
\hline \multirow[t]{2}{*}{ Parameters } & \multirow{2}{*}{$\begin{array}{c}\text { Unaffected cases } \\
(\mathrm{N}=20)\end{array}$} & \multirow{2}{*}{$\begin{array}{c}\text { Affected cases } \\
(\mathrm{N}=51)\end{array}$} & \multirow[t]{2}{*}{$P$ value } \\
\hline & & & \\
\hline Age (years) & $39.5(32.3-52.8)$ & $46.0(36.0-54.0)$ & 0.297 \\
\hline Female & $11(55.0 \%)$ & $37(72.6 \%)$ & \\
\hline Male & $9(45.0 \%)$ & $14(27.4 \%)$ & \\
\hline BMI $\left(\mathrm{kg} / \mathrm{m}^{2}\right)$ & $23.1(20.8-23.9)$ & $30.9(27.3-35.1)$ & $<0.001$ \\
\hline Weight (kg) & $56.9(51.6-61.9)$ & $79.0(67.1-85.3)$ & $<0.001$ \\
\hline Height (cm) & $157.8(152.1-164.4)$ & $157.0(152.0-164.8)$ & 0.990 \\
\hline Waist circumference $(\mathrm{cm})$ & $79.5(74.2-84.5)$ & $97.0(91.0-103.1)$ & $<0.001$ \\
\hline Fasting glucose (mg/dL) & $88(85-93)$ & $95(89-117)$ & 0.007 \\
\hline $\mathrm{HbA1c}(\%)$ & $5.6(5.3-5.9)$ & $6.1(5.7-7.2)$ & 0.001 \\
\hline Fasting insulin $(\mu \mathrm{U} / \mathrm{mL})$ & $5.6(3.5-10.2)$ & $11.9(9.0-20.6)$ & $<0.001$ \\
\hline HOMA-IR & $1.33(0.74-2.21)$ & $2.74(2.74-3.64)$ & $<0.001$ \\
\hline Total cholesterol (mg/dL) & $193(170-220)$ & $204(182-227)$ & 0.354 \\
\hline LDL-C (mg/dL) & $111(95-142)$ & $122(105-148)$ & 0.357 \\
\hline HDL-C (mg/dL) & $64(55-75)$ & $49(43-60)$ & $<0.001$ \\
\hline Triglyceride (mg/dL) & $63(53-92)$ & $133(104-171)$ & $<0.001$ \\
\hline$\alpha-M S H(p g / m L)$ & $36.3(17.1-75.8)$ & $31.4(18.3-43.9)$ & 0.312 \\
\hline Systolic blood pressure $(\mathrm{mmHg})$ & $116(106-142)$ & $134(124-148)$ & 0.005 \\
\hline Diastolic blood pressure (mmHg) & $74(65-86)$ & $78(72-85)$ & 0.094 \\
\hline
\end{tabular}

$\mathrm{BMI}=$ body mass index; HbA1c = hemoglobin A1c; HOMA-IR = homeostatic model assessment for insulin resistance; LDL-C = low-density lipoprotein cholesterol; HDL-C = high-density lipoprotein cholesterol; TG = triglyceride; $\alpha$-MSH = serum alpha-melanocyte-stimulating hormone. Data are reported as median (IQR); P value $<0.05$ was considered to be statistically significant.

\section{Probands}

There were four female probands and seven male probands between the ages of 9 and 19. Almost all probands were severely to morbidly obese with a history of early-onset obesity. Two probands had type 2 diabetes with HbA1c above $7 \%$. Low HDL-C levels (HDL-C $<40 \mathrm{mg} / \mathrm{dL}$ ) were common among probands. A markedly elevated $\alpha-\mathrm{MSH}$ level was found in one proband (Family K). 


\section{Affected and unaffected family members}

The affected family members were significantly heavier $(P<0.001)$, had a higher BMI $(P<0.001)$, and a greater WC $(P<0.001)$ than the unaffected family members. Affected family members also had a significantly higher systolic blood pressure $(P=0.005)$, fasting blood glucose $(P=0.007), \operatorname{HbA1c}(P=0.001)$, insulin $(P<0.001), \mathrm{HOMA}-\mathrm{IR}(P<0.001), \mathrm{TG}(P<0.001)$, and lower HDL-C $(P<0.001)$ than the unaffected family members. Levels of $\alpha-M S H$ were not significantly different between the two groups.

\section{MC3R gene analysis}

Analysis of the MC3R gene in probands and their family members did not reveal any novel mutations. Two previously described single nucleotide polymorphisms (SNPs) were detected: rs3746619 (C/A), and rs3827103 (G/A) as a haplotype block. A total of three probands, 11 affected subjects, and four unaffected subjects carried the heterozygous variants of these two common SNPs (Table 4). Analysis of these SNPs in the 100 healthy controls found that 10 were homozygous variant carriers, and 44 were heterozygous carriers.

\begin{tabular}{|c|c|c|c|c|c|}
\hline \multirow[t]{2}{*}{ Family } & \multirow[t]{2}{*}{ Subject types } & \multicolumn{2}{|c|}{ MC3R (rs3746619 and rs3827103)a } & \multicolumn{2}{|c|}{ MC4R variations ${ }^{b}$} \\
\hline & & Wt & Hetero & $\mathrm{Wt}$ & Hetero \\
\hline \multirow[t]{3}{*}{$A$} & Proband $(\mathrm{N}=1)$ & 1 & & 1 & \\
\hline & Affected case $(\mathrm{N}=11)$ & 10 & 1 & 11 & \\
\hline & Unaffected case $(\mathrm{N}=4)$ & 2 & 2 & 4 & \\
\hline \multirow[t]{3}{*}{ B } & Proband $(\mathrm{N}=1)$ & & 1 & 1 & \\
\hline & Affected case $(\mathrm{N}=2)$ & 1 & 1 & 2 & \\
\hline & Unaffected case $(\mathrm{N}=0)$ & & & & \\
\hline \multirow[t]{3}{*}{ C } & Proband $(\mathrm{N}=1)$ & 1 & & 1 & \\
\hline & Affected case $(\mathrm{N}=3)$ & 2 & 1 & 3 & \\
\hline & Unaffected case $(\mathrm{N}=2)$ & 2 & & 2 & \\
\hline \multirow[t]{3}{*}{$\mathrm{D}$} & Proband $(N=1)$ & 1 & & & 1 (rs34114122) \\
\hline & Affected case $(\mathrm{N}=4)$ & 2 & 2 & 3 & 1 (rs34114122) \\
\hline & Unaffected case $(\mathrm{N}=1)$ & 1 & & & 1 (rs34114122) \\
\hline \multirow[t]{3}{*}{ E } & Proband $(\mathrm{N}=1)$ & 1 & & 1 & \\
\hline & Affected case $(N=21)$ & 18 & 3 & 21 & \\
\hline & Unaffected case $(\mathrm{N}=8)$ & 6 & 2 & 8 & \\
\hline \multirow[t]{3}{*}{$\mathrm{F}$} & Proband $(\mathrm{N}=1)$ & 1 & & & 1 (rs182455344) \\
\hline & Affected case $(\mathrm{N}=2)$ & 1 & 1 & 1 & 1 (rs182455344) \\
\hline & Unaffected case $(\mathrm{N}=0)$ & & & & \\
\hline \multirow[t]{3}{*}{ G } & Proband $(\mathrm{N}=1)$ & 1 & & 1 & \\
\hline & Affected case $(\mathrm{N}=2)$ & 1 & 1 & 2 & \\
\hline & Unaffected case $(\mathrm{N}=2)$ & 2 & & 2 & \\
\hline \multirow[t]{3}{*}{$\mathrm{H}$} & Proband $(\mathrm{N}=1)$ & & 1 & 1 & \\
\hline & Affected case $(N=1)$ & 1 & & 1 & \\
\hline & Unaffected case $(\mathrm{N}=0)$ & & & & \\
\hline \multirow[t]{3}{*}{ । } & Proband $(N=1)$ & 1 & & 1 & \\
\hline & Affected case $(\mathrm{N}=2)$ & 2 & & 2 & \\
\hline & Unaffected case $(\mathrm{N}=1)$ & 1 & & 1 & \\
\hline \multirow[t]{3}{*}{$\mathrm{J}$} & Proband $(\mathrm{N}=1)$ & & 1 & & 1 (rs61741819) \\
\hline & Affected case $(\mathrm{N}=2)$ & 1 & 1 & 1 & 1 (rs61741819) \\
\hline & Unaffected case $(\mathrm{N}=0)$ & & & & \\
\hline \multirow{3}{*}{ K } & Proband $(\mathrm{N}=1)$ & 1 & & 1 & \\
\hline & Affected case $(\mathrm{N}=1)$ & 1 & & 1 & \\
\hline & Unaffected case $(\mathrm{N}=2)$ & 2 & & 2 & \\
\hline
\end{tabular}

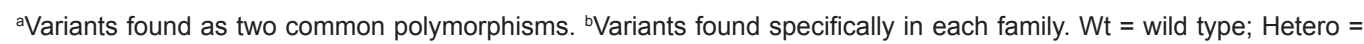
heterozygous carrier. 


\section{MC4R gene analysis}

Analysis of the MC4R gene revealed three different nucleotide sequence variants in probands and family members of family D, F, and J; rs34114122 (A/C), rs182455344 (T/C), and rs61741819 (C/T) (Table 4). All variant carriers were heterozygous. The rs34114122 and rs61741819 variants have been previously reported, but rs182455344 was novel.

\section{rs34114122 (A/C)}

Proband D was an 11-year-old boy who was morbidly obese (percentage weight for height $243 \%$, BMI $\left.47.6 \mathrm{~kg} / \mathrm{m}^{2}\right)$. He had a large birth weight $(4.800 \mathrm{~kg})$ and had been severely obese since the age of 1 . Fasting blood glucose was normal, but he had marked insulin resistance (HOMA-IR of 13.79). The one unaffected and four affected family members of proband $D$ were his father (BMI $24.6 \mathrm{~kg} / \mathrm{m}^{2}$ ), his paternal grandmother (BMI $\left.25.3 \mathrm{~kg} / \mathrm{m}^{2}\right)$, his mother (BMl $46 \mathrm{~kg} /$ $\mathrm{m}^{2}$ ), his aunt (BMI $\left.34.3 \mathrm{~kg} / \mathrm{m}^{2}\right)$, and his maternal grandmother (BMI $\left.33.1 \mathrm{~kg} / \mathrm{m}^{2}\right)$. A heterozygous substitution of nucleotide from A to $C$ at position 242 of the MC4R gene was found in the proband, his father, and his paternal grandmother. This variant was located in the 5'-UTR of the MC4R gene, rs34114122 (Table 5 and Figure 1), which was the location of transcriptional site area. This variant was passed from grandmother to father, and to proband, however his father was borderline overweight (BMI $\left.24.6 \mathrm{~kg} / \mathrm{m}^{2}\right)$. Yet, all three carriers in this family had a history of earlyonset obesity before 5 years of age, and had been hyperphagic since childhood. In addition, the proband had severe hyperinsulinemia, and his father had a high level of a-MSH (Table 6). The MAF was $4.4 \%$ for the overall population (data from HapMap project). One hundred healthy control subjects were also analyzed for allele frequency and it was found that three of them were heterozygous carriers of the variant $(1.5 \%)$.

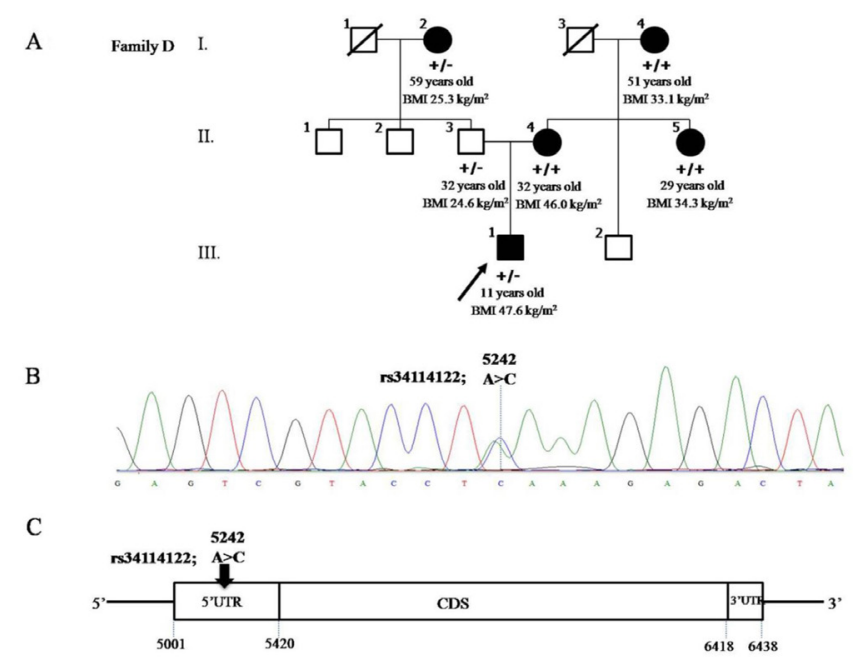

Figure 1. rs34114122 variant in the MC4R gene of family D. A. Pedigree of family D showing genotype of rs34114122 in the MC4R gene. B. Electropherograms showing the transition of nucleotide in rs34114122. C. Schematic representation of the MC4R gene showing the location of rs34114122 in the MC4R gene. Squares and circles represent males and females, respectively; dark-shaded figures denote family members with overweight or obesity; arrows indicate probands; symbols indicate genotype +/+ wild-type allele; +/- heterozygous allele. 


\begin{tabular}{|c|c|c|c|c|c|c|c|c|c|c|c|}
\hline Gene & Family & $\begin{array}{l}\text { Portion } \\
\text { of gene }\end{array}$ & $\begin{array}{c}\text { Nucleotide } \\
\text { change }\end{array}$ & $\begin{array}{c}\text { Codon } \\
\text { change }\end{array}$ & $\begin{array}{c}\text { Amino acid } \\
\text { change }\end{array}$ & SNP ID & $\begin{array}{c}\text { No. of } \\
\text { subject }\end{array}$ & $\begin{array}{c}\text { Allele } \\
\text { frequency }\end{array}$ & $\begin{array}{c}\text { European } \\
\text { MAF }^{\mathrm{b}}\end{array}$ & $\begin{array}{c}\text { African } \\
\text { MAF }^{\mathrm{b}}\end{array}$ & $\begin{array}{l}\text { Asian } \\
\text { MAFb }^{\mathrm{b}}\end{array}$ \\
\hline \multirow[t]{3}{*}{ MC4R } & $\mathrm{D}$ & 5' UTR & g. $5242 A>C$ & - & - & rs34114122 & 3 & $1.5 \%$ & 0.01 & 0.11 & 0.05 \\
\hline & $\mathrm{F}$ & near 3' & g. $6460 \mathrm{~T}>\mathrm{C}$ & - & - & rs182455344 & 2 & not found & not found & not found & 0.01 \\
\hline & $\mathrm{J}$ & CDS & g. $6013 \mathrm{C}>\mathrm{T}$ & ATC $>$ ATT & Ile198Ile & rs61741819 & 2 & not found & not found & 0.04 & not found \\
\hline
\end{tabular}

UTR = untranslated region; CDS = coding DNA sequence; $g$. = genomic; lle = isoleucine; SNP = single nucleotide

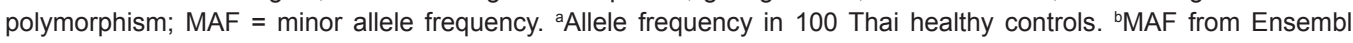
database.

\begin{tabular}{|c|c|c|c|c|c|c|c|c|c|c|c|c|}
\hline \multicolumn{2}{|c|}{ Family } & \multirow{2}{*}{$\begin{array}{l}\text { Onset obesity } \\
\text { age (years) } \\
<1\end{array}$} & \multirow{2}{*}{$\begin{array}{c}\begin{array}{c}\mathrm{BMI} \\
\left(\mathrm{kg} / \mathrm{m}^{2}\right)\end{array} \\
47.6\end{array}$} & \multirow{2}{*}{$\begin{array}{l}\begin{array}{l}W C \\
(\mathrm{~cm})\end{array} \\
130\end{array}$} & \multirow{2}{*}{$\begin{array}{c}\begin{array}{c}\text { Fasting } \\
\text { glucose }(\mathrm{mg} / \mathrm{dL})\end{array} \\
79\end{array}$} & \multirow{2}{*}{$\begin{array}{c}\mathrm{HbA1c} \\
(\%)\end{array}$} & \multirow{2}{*}{$\begin{array}{c}\begin{array}{c}\text { Fasting } \\
\text { insulin }(\mu \mathrm{U} / \mathrm{mL})\end{array} \\
70.7\end{array}$} & \multirow{2}{*}{$\begin{array}{c}\text { HOMA-IR } \\
13.79\end{array}$} & \multirow{2}{*}{$\begin{array}{c}\begin{array}{c}\text { LDL-C } \\
(\mathrm{mg} / \mathrm{dL})\end{array} \\
103\end{array}$} & \multirow{2}{*}{$\begin{array}{c}\begin{array}{c}\mathrm{HDL}-\mathrm{C} \\
(\mathrm{mg} / \mathrm{dL})\end{array} \\
55\end{array}$} & \multirow{2}{*}{$\begin{array}{c}\begin{array}{c}\mathrm{TG} \\
(\mathrm{mg} / \mathrm{dL})\end{array} \\
57\end{array}$} & \multirow{2}{*}{$\begin{array}{c}\begin{array}{c}\alpha-M S H \\
(\mathrm{pg} / \mathrm{mL})\end{array} \\
40.3\end{array}$} \\
\hline D & Proband & & & & & & & & & & & \\
\hline & His father & $<1$ & 24.6 & 84 & 77 & 5.5 & 2.94 & 0.56 & 94 & 66 & 49 & 485.9 \\
\hline & His grandmother & $<5$ & 25.3 & 96 & 92 & 5.7 & 11.65 & 2.65 & 73 & 67 & 153 & 55.3 \\
\hline \multirow[t]{2}{*}{$\mathrm{F}$} & Proband & 4 & 27.3 & 90 & 78 & 5.9 & 24.6 & 4.74 & 101 & 60 & 69 & 20.0 \\
\hline & His mother & $<5$ & 35.2 & 103 & 123 & 8.2 & 28.8 & 8.75 & 46.2 & 55 & 104 & 48.1 \\
\hline \multirow[t]{2}{*}{$\mathrm{J}$} & Proband & 2 & 42.5 & 118 & 88 & 5.9 & 40.9 & 8.88 & 107 & 36 & 122 & 24.2 \\
\hline & His mother & $<5$ & 39.5 & 122 & 105 & 5.8 & 17.32 & 4.49 & 163 & 49 & 191 & 48.8 \\
\hline
\end{tabular}

$\mathrm{BMI}=$ body mass index; $\mathrm{WC}=$ waist circumference; HbA1c = hemoglobin A1c; HOMA-IR = homeostatic model assessment for insulin resistance; LDL-C = low-density lipoprotein cholesterol; HDL-C = high-density; TG = triglyceride; a-MSH = serum alpha-melanocyte-stimulating hormone.

\section{rs182455344 (T/C)}

This male proband $\mathrm{F}$ was 13 years old, and his percentage weight for height was $151 \%$ (BMI $\left.27.3 \mathrm{~kg} / \mathrm{m}^{2}\right)$. He had a low birth weight of $2.070 \mathrm{~kg}$ and started gaining weight by age 4 . Although he had a normal fasting blood glucose and $\mathrm{HbA} 1 \mathrm{c}$, his insulin level was high, indicating insulin resistance. His elder sister and mother were affected, subjects with a BMl of 28.3 and $35.2 \mathrm{~kg} / \mathrm{m}^{2}$, respectively. DNA analysis of the MC4R gene found one variant identified as a heterozygous nucleotide substitution, from T to $C$ at the downstream segment the MC4R gene, which was near 3'-UTR - rs182455344 (Table 5) found in the proband of family $F$ and his mother (Figure 2). Both variant carriers had a history of earlyonset obesity before age 5 (Table 6 ). The MAF was $0.2 \%$ in overall population (data from HapMap project). This mutation was not found in any of the 100 healthy control subjects.

\section{rs61741819 (C/T)}

Proband $\mathrm{J}$ was a 12-year-old morbidly obese boy (percentage weight for height $240 \%$, BMI $42.5 \mathrm{~kg} / \mathrm{m}^{2}$ ). He had a normal birth weight but gained weight rapidly since he was 2 years old. His father and mother were both obese with a BMI of 32.4 and $39.5 \mathrm{~kg} / \mathrm{m}^{2}$, respectively. Sequencing of the MC4R gene found one variant in proband $\mathrm{J}$ and his mother (Figure 3), in the form of a heterozygous substitution of nucleotide from $\mathrm{C}$ to $\mathrm{T}$ at codon 198 of the coding region indicated as rs61741819 (Table 5). This variant is synonymous with the single nucleotide polymorphism, Ile198Ile. The variant carriers had a history of early-onset obesity before age 5 . The MAF was $1.01 \%$ of the overall population (data from HapMap project); however, this variant was not found in any of the 100 healthy control subjects. 
A

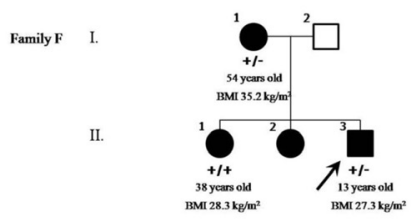

B

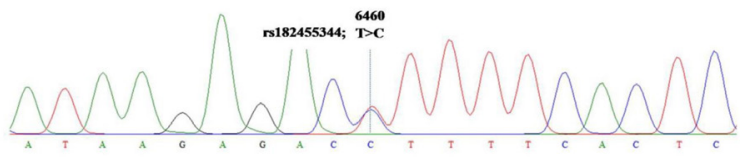

C

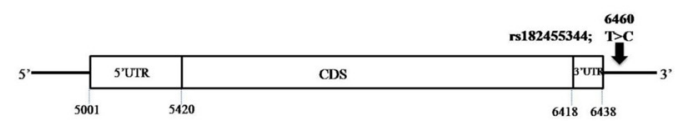

Figure 2. rs 182455344 variant in the MC4R gene of family F. A. Pedigree of family F showing genotype of rs 182455344 in the MC4R gene. B. Electropherograms showing the transition of nucleotide in rs 182455344 . C. Schematic representation of the MC4R gene showing the location of rs182455344 in the MC4R gene. Squares and circles represent males and females, respectively; dark-shaded figures denote family members with overweight or obesity; arrows indicate probands; symbols indicate genotype +/+ wild-type allele; +/- heterozygous allele.

A

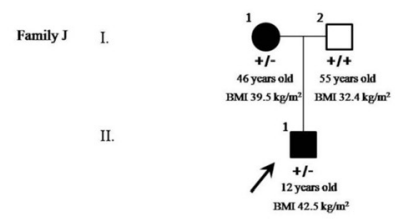

B

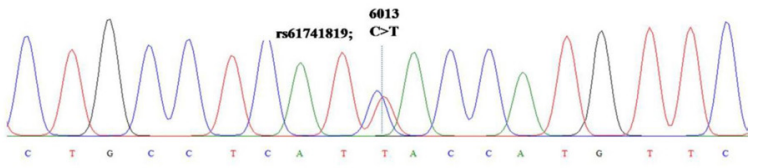

$\mathrm{C}$

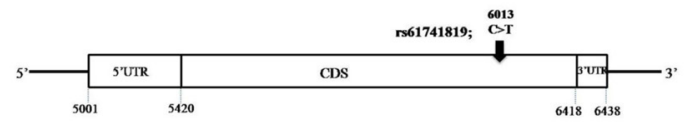

Figure 3. rs61741819 variant in the MC4R gene of family J. A. Pedigree of family J showing genotype of rs61741819 in the MC4R gene. B. Electropherograms showing the transition of nucleotide in rs61741819. C. Schematic representation of the MC4R gene showing the location of rs61741819 in the MC4R gene. Squares and circle represent males and female, respectively; dark-shaded figures denote family members with overweight or obesity; arrows indicate probands; symbols indicate genotype +/+ wild-type allele; +/- heterozygous allele".

\section{DISCUSSION}

In this study, the authors searched for variations of the MC3R and MC4R genes in 11 earlyonset obese Thai children and their family members, to identify possible monogenic causes of obesity. Three different MC4R variants were presented in three families, and two common polymorphisms of the MC3R gene were found in nine families. All variants were found in a heterozygous state.

The first SNP, rs34114122 (A/C), was located at the 5'-UTR of the promoter site in the MC4R gene and has been previously reported in European, American, and Asian populations 
(Jacobson et al., 2002; Lubrano-Berthelier et al., 2003; Stutzmann et al., 2007; Van Den Berg et al., 2011; Tan et al., 2014). This variant was not found to be associated with obesity. However, other variants of the MC4R within the 5'-UTR were found to have a negative impact on MC4R function (Tan et al., 2014). Despite being outside of the coding region of the gene, variants in the 5'-UTR may interfere with the process of protein synthesis due to its location in the transcriptional site. Protein synthesis involves multiple complex steps including transcription, mRNA stability, translation, posttranslational modification, protein folding, and various error-checking mechanisms. A single point mutation of a nucleotide may affect many regulatory steps involved in protein synthesis. From previous studies, SNPs in the 5'-UTR have been found to cause some diseases, such as the GDF5 gene associated with osteoarthritis (Mu et al., 2014) and the HOPX gene related to syncope in hypertrophic cardiomyopathy patients (Gulec et al., 2014).

In this study, rs34114122 was found in the proband of Family D, his father, and his paternal grandmother, indicating an autosomal dominant inheritance. Although his father was nearly overweight and his grandmother was mildly obese, this might be caused by an incomplete penetrance. A study from the UK found that there was an age-related decrease in the characteristics of MC4R deficiency (Farooqi et al., 2003a). All three carriers had history of early-onset obesity before 5 years of age, however only the father had a high level of $\alpha-\mathrm{MSH}$. It might result from some defective functions of the MC4R protein or it might be from haploinsufficiency. Perhaps, other genetic factors may have been inherited from the mother since his mother was severely obese. Although previous studies found rs34114122 in both obese and normal-weight people, we determined the inheritance of this variant in this study. Therefore, a functional study of this variant will be very useful to identify its genetic influence on obesity. This point mutation was found in 3 of the 100 healthy Thai controls accounting for $1.5 \%$, however MAF has been found in $4.4 \%$ of the overall population (data from HapMap project).

The rs61741819 variant at codon 198 within the fifth transmembrane domain of MC4R, which is a 7-transmembrane G-protein-coupled receptor, is a heterozygous nucleotide substitution from $\mathrm{C}$ to T resulting in no change of isoleucine (Ile198lle). There have been three previous reports of this silent variation. The first report found only one heterozygous carrier out of 4068 representative population-based samples from a KORA-S4 adult study group in Germany (Hinney et al., 2006). In Austria, one obese mother of obese children was identified as a heterozygous carrier of this silent variation (Rettenbacher et al., 2007). In the Netherlands, four heterozygous carriers and one homozygous carrier were reported among 291 obese children (Van Den Berg et al., 2011). Those reports have not found an association of this silent variant with obesity phenotypes. Silent mutations result in no change of the amino acid. There are no reports about functional studies in this variation, and it is not known whether the silent mutation might cause an altered expression of receptor genes, as shown in many studies regarding silent mutation of receptor genes (Duan et al., 2003; Igreja et al., 2010). In addition, the silent mutation might affect the protein's primary, secondary, or tertiary structures by affecting splicing accuracy, translation fidelity, conformation, and function of proteins (Komar, 2007). This mutation was found in a 12-year-old male proband and his 46-year-old mother in family $\mathrm{J}$. His mother may have passed this heterozygous variant to the proband showing an autosomal dominant inheritance with complete penetrance since both exhibited extreme obesity. This point mutation was not found in 100 healthy Thai controls; however, MAF suggests $1.01 \%$ prevalence in overall population (data from HapMap project).

Lastly, the rs182455344 variant located near the 3'-UTR of MC4R gene resulted from a T to $C$ nucleotide substitution. This SNP was located 42 bp after the translation stop codon and 126 bp before the polyadenylation signal (Van Den Berg et al., 2011). This SNP has not been previously 
associated with obesity. It was found in the proband and his mother in family F, showing autosomal dominant inheritance. This SNP was not detected in any of the 100 healthy Thai controls, and MAF occurs in $0.2 \%$ overall population (data from HapMap project). Even though this variant is not within the coding region, there were strong associations between rs17782313 mapped $188 \mathrm{~kb}$ downstream of the MC4R to fat mass, weight, and obesity risk (Loos et al., 2008). It is plausible that variants near the 3 ' locus might influence post-transcriptional gene expression, as suggested by a functional experiment (Liu et al., 2012). Together with the $3^{\prime}$ end variants, these have medical relevance by deregulating mRNA maturation (Danckwardt et al., 2008).

In terms of MC3R variants, two previously known common polymorphisms have been detected: rs3746619 and rs3827103 as a haplotype block. This study found these SNPs in 3 of 11 probands, 11 of 51 affected subjects, and 4 of 20 unaffected subjects with heterozygous state. There were no associations found with any anthropometric parameters in this study (data not shown). Therefore, they are likely represented as common polymorphisms. Moreover, 54 of 100 Thai healthy controls were found to have the two common variants, 10 of which had homozygous carriers accounting for allele frequency around $32 \%$. MAF occurs in $20 \%$ of the overall population (data from HapMap project), and there is a discrepant variety in different ethnicities.

Our study found that 3 of $11(27 \%)$ probands with early-onset obesity before age 5 had three distinct variations of the MC4R gene. These variants did not cause changes of amino acid sequence. All variants were dominantly inherited in a heterozygous state with an inconsistent penetrance and variable expressivity observed between families. This might be due to the difference in defective functions of MC4R variations and could also be influenced by environmental factors. Many studies indicate that the inheritance patterns of MC4R variants vary considerably between families, and exhibit either dominant or recessive patterns (Hinney et al., 1999; Farooqi et al., 2000). Some recent studies suggest a co-dominant pattern of inheritance (Kobayashi et al., 2002; Lee, 2012). Moreover, all MC4R variant carriers had a history of early-onset obesity before age 5 , and most of them were found to have higher insulin levels than other obese wild type participants. A previous study showed that the a-MSH levels of MC4R mutant carriers was significantly higher than that of wild type obese subjects (Roth et al., 2010). The lack of genetic information in other family members, especially those who have a history of obesity, is our limitation. Further analysis is required to reveal the functions of these variants and a large-scale study is warranted.

In conclusion, this is the first family-based study to screen for MC3R and MC4R variations in early-onset obese Thai children and their family members. Two common MC3R polymorphisms, rs3746619 and rs3827103, were detected among probands, unaffected, and affected family members. Three distinct variants of MC4R, rs34114122, rs61741819, and rs182455344, were found in 3 probands with autosomal dominant inheritance. These variants could possibly contribute to a genetic influence of early-onset obesity in Thais. Although previous reports have shown no associations of these three variants with obesity, most other studies were done using European and American populations. There has not been much research performed in Asia, especially in Thai populations. Furthermore, variant screening of the MC4R gene might reveal a cause of inherited obesity. In the future, this gene should be considered a genetic maker to identify a high risk of obesity in Thais.

\section{Conflicts of interest}

The authors declare no conflict of interest. 


\section{ACKNOWLEDGMENTS}

Research supported by the Thailand Research Fund and Mahidol University through the RGJ-Ph.D. Program (Grant \#PHD/0366/2550), the Research Fund of Mahidol University, and an NRU grant from the Center for Biopharmaceutical Development \& Innovative Therapy, Mahidol University, Thailand. The authors would like to thank all participating children and their families, and the staff of the Department of Tropical Nutrition and Food Science, Faculty of Tropical Medicine; the Department of Pediatrics, Faculty of Medicine Siriraj Hospital; the Department of Pediatrics, Faculty of Medicine Ramathibodi Hospital; and the Division of Molecular Genetics, Department of Research \& Development, Faculty of Medicine Siriraj Hospital, Mahidol University for their cooperation and extensive assistance.

\section{REFERENCES}

Calton MA, Ersoy BA, Zhang S, Kane JP, et al. (2009). Association of functionally significant Melanocortin-4 but not Melanocortin-3 receptor mutations with severe adult obesity in a large North American case-control study. Hum. Mol. Genet. 18: 1140-1147.

Chen AS, Marsh DJ, Trumbauer ME, Frazier EG, et al. (2000). Inactivation of the mouse melanocortin-3 receptor results in increased fat mass and reduced lean body mass. Nat. Genet. 26: 97-102.

Chuenta W, Phonrat B, Tungtrongchitr A, Limwongse C, et al. (2015). Common variations in the FTO gene and obesity in Thais: a family-based study. Gene 558: 75-81.

Danckwardt S, Hentze MW and Kulozik AE (2008). 3' end mRNA processing: molecular mechanisms and implications for health and disease. EMBO J. 27: 482-498.

Duan J, Wainwright MS, Comeron JM, Saitou N, et al. (2003). Synonymous mutations in the human dopamine receptor D2 (DRD2) affect mRNA stability and synthesis of the receptor. Hum. Mol. Genet. 12: 205-216.

Farooqi IS and O'rahilly S (2008). Mutations in ligands and receptors of the leptin-melanocortin pathway that lead to obesity. Nat. Clin. Pract. Endocrinol. Metab. 4: 569-577.

Farooqi IS, Yeo GS, Keogh JM, Aminian S, et al. (2000). Dominant and recessive inheritance of morbid obesity associated with melanocortin 4 receptor deficiency. J. Clin. Invest. 106: 271-279.

Farooqi IS, Keogh JM, Yeo GS, Lank EJ, et al. (2003a). Clinical spectrum of obesity and mutations in the melanocortin 4 receptor gene. N. Engl. J. Med. 348: 1085-1095.

Farooqi IS, Yeo GS and O'rahilly S (2003b). Binge eating as a phenotype of melanocortin 4 receptor gene mutations. N. Engl. J. Med. 349: 606-609.

Gulec C, Abaci N, Bayrak F, Bayrak EK, et al. (2014). Association between non-coding polymorphisms of HOPX gene and syncope in hypertrophic cardiomyopathy. Anadolu. Kardiyol. Derg. 14: 617-624.

Hinney A, Schmidt A, Nottebom K, Heibult O, et al. (1999). Several mutations in the melanocortin-4 receptor gene including a nonsense and a frameshift mutation associated with dominantly inherited obesity in humans. J. Clin. Endocrinol. Metab. 84: 1483-1486.

Hinney A, Bettecken T, Tarnow P, Brumm H, et al. (2006). Prevalence, spectrum, and functional characterization of melanocortin-4 receptor gene mutations in a representative population-based sample and obese adults from Germany. J. Clin. Endocrinol. Metab. 91: 1761-1769.

Hinney A, Volckmar AL and Knoll N (2013). Melanocortin-4 receptor in energy homeostasis and obesity pathogenesis. Prog. Mol. Biol. Transl. Sci. 114: 147-191.

Hjelmborg J, Fagnani C, Silventoinen K, Mcgue M, et al. (2008). Genetic influences on growth traits of BMI: a longitudinal study of adult twins. Obesity (Silver Spring) 16: 847-852.

Igreja S, Chahal HS, King P, Bolger GB, et al. (2010). Characterization of aryl hydrocarbon receptor interacting protein (AIP) mutations in familial isolated pituitary adenoma families. Hum. Mutat. 31: 950-960.

Jacobson P, Ukkola O, Rankinen T, Snyder EE, et al. (2002). Melanocortin 4 receptor sequence variations are seldom a cause of human obesity: the Swedish Obese Subjects, the HERITAGE Family Study, and a Memphis cohort. J. Clin. Endocrinol. Metab. 87: 4442-4446.

Kobayashi H, Ogawa Y, Shintani M, Ebihara K, et al. (2002). A Novel homozygous missense mutation of melanocortin-4 receptor (MC4R) in a Japanese woman with severe obesity. Diabetes 51: 243-246.

Komar AA (2007). Silent SNPs: impact on gene function and phenotype. Pharmacogenomics 8: 1075-1080. 
Lee JH, Reed DR and Price RA (1997). Familial risk ratios for extreme obesity: implications for mapping human obesity genes. Int. J. Obes. Relat. Metab. Disord. 21: 935-940.

Lee YS (2009). The role of leptin-melanocortin system and human weight regulation: lessons from experiments of nature. Ann. Acad. Med. Singapore 38: 34-11.

Lee YS (2012). Melanocortin 3 receptor gene and melanocortin 4 receptor gene mutations: the Asian perspective. Diabetes Metab. Res. Rev. 28 (Suppl 2): 26-31.

List JF and Habener JF (2003). Defective melanocortin 4 receptors in hyperphagia and morbid obesity. N. Engl. J. Med. 348: 1160-1163.

Liu M, Wang L, Bongartz T, Hawse JR, et al. (2012). Aromatase inhibitors, estrogens and musculoskeletal pain: estrogendependent T-cell leukemia 1A (TCL1A) gene-mediated regulation of cytokine expression. Breast Cancer Res. 14: R41.

Loos RJ, Lindgren CM, Li S, Wheeler E, et al. (2008). Common variants near MC4R are associated with fat mass, weight and risk of obesity. Nat. Genet. 40: 768-775.

Lubrano-Berthelier C, Cavazos M, Le Stunff C, Haas K, et al. (2003). The human MC4R promoter: characterization and role in obesity. Diabetes 52: 2996-3000.

Mencarelli M, Dubern B, Alili R, Maestrini S, et al. (2011). Rare melanocortin-3 receptor mutations with in vitro functional consequences are associated with human obesity. Hum. Mol. Genet. 20: 392-399.

Mu J, Ge W, Zuo X, Chen Y, et al. (2014). A SNP in the 5'-UTR of GDF5 is associated with susceptibility to symptomatic lumbar disc herniation in the Chinese Han population. Eur. Spine J. 23: 498-503.

$\mathrm{Ng} \mathrm{M}$, Fleming T, Robinson M, Thomson B, et al. (2014). Global, regional, and national prevalence of overweight and obesity in children and adults during 1980-2013: a systematic analysis for the Global Burden of Disease Study 2013. Lancet 384: 766-781.

Oswal A and Yeo GS (2007). The leptin melanocortin pathway and the control of body weight: lessons from human and murine genetics. Obes. Rev. 8: 293-306.

Rankinen T and Bouchard C (2006). Genetics of food intake and eating behavior phenotypes in humans. Annu. Rev. Nutr. 26: 413-434.

Rettenbacher E, Tarnow P, Brumm H, Prayer D, et al. (2007). A novel non-synonymous mutation in the melanocortin-4 receptor gene (MC4R) in a 2-year-old Austrian girl with extreme obesity. Exp. Clin. Endocrinol. Diabetes 115: 7-12.

Roth CL, Enriori PJ, Gebhardt U, Hinney A, et al. (2010). Changes of peripheral alpha-melanocyte-stimulating hormone in childhood obesity. Metabolism 59: 186-194.

Santini F, Maffei M, Pelosini C, Salvetti G, et al. (2009). Melanocortin-4 receptor mutations in obesity. Adv. Clin. Chem. 48: 95-109.

Schwartz MW, Woods SC, Porte D Jr., Seeley RJ, et al. (2000). Central nervous system control of food intake. Nature 404: 661-671.

Stutzmann F, Vatin V, Cauchi S, Morandi A, et al. (2007). Non-synonymous polymorphisms in melanocortin-4 receptor protect against obesity: the two facets of a Janus obesity gene. Hum. Mol. Genet. 16: 1837-1844.

Tan KM, Ooi SQ, Ong SG, Kwan CS, et al. (2014). Functional characterization of variants in MC4R gene promoter region found in obese children. J. Clin. Endocrinol. Metab. 99: E931-935.

Vaisse C, Clement K, Guy-Grand B and Froguel P (1998). A frameshift mutation in human MC4R is associated with a dominant form of obesity. Nat. Genet. 20: 113-114.

Van Den Berg L, Van Beekum O, Heutink P, Felius BA, et al. (2011). Melanocortin-4 receptor gene mutations in a Dutch cohort of obese children. Obesity (Silver Spring) 19: 604-611.

Yamborisut U and Mo-Suwan L (2014). Prevalence of childhood and adolescent obesity in Thailand: a review. J. Med. Assoc. Thai. 97: 44-51.

Yeo GS, Farooqi IS, Aminian S, Halsall DJ, et al. (1998). A frameshift mutation in MC4R associated with dominantly inherited human obesity. Nat. Genet. 20: 111-112. 